\title{
Association of CD14 -159 Gene Polymorphism with Characteristics and Outcome of Diffuse Large B-cell Lymphomas
}

\author{
Lavinika ATANASKOVIC ${ }^{1}$, Olivera TARABAR ${ }^{1}$, Zvonko MAGIC ${ }^{2}$, \\ Dragana STAMATOVIC ${ }^{1}$, Andjelina ZIVANOVIC ${ }^{1}$, Slobodan MARJANOVIC ${ }^{1}$, \\ Bojana CIKOTA-ALEKSIC ${ }^{2}$ \\ ${ }^{1}$ Military Medical Academy, Clinic of Hematology \\ ${ }^{2}$ Military Medical Academy, Institute of Medical Research, Belgrade, SERBIA
}

\begin{abstract}
The aim of this study was to assess the association between CD14 -159 genotypes and DLBCL characteristics, clinical course and outcome, respectively. The study included 114 patients with the newly diagnosed DLBCL. All patients received R-CHOP therapy (6-8 cycles). CD14 -159C $\rightarrow$ T genotyping was performed by using PCR RFLP. Obtained frequencies of CD14 -159 genotypes were as follows: $23 \%$ of CC, $41 \%$ of CT and $36 \%$ of $\Pi$. Statistically significant association between CD14 genotypes and clinical characteristics (age, sex, disease stage, extranodal sites, bulky disease, IPI, lymphocyte and lymphocyte/monocyte count) was found only for extranodal disease $(p=0.01)$. CT/TT carriers more frequently presented extranodal disease (OR 3.191, 95\% Cl: 1.282-7.94). In addition, these patients had higher pretreatment values of CRP $(p=0.078)$. During the therapy, -infections $(p=0.083)$, and earlytreatment-related complications $(\mathrm{p}=0.079)$ were more commonly present in carriers of CC genotype. In patients with extranodal DLBCL, TT genotype was associated with superior OS $(p=0.049)$ and RFS, $(p=0.018)$. Multivariate analysis revealed IPI (HR 2.422, 95\% Cl 1.114-5.264; $p=0.026$ ) and CD14 TT genotype (HR 0.503, 95\% Cl: $0.262-0.963 ; p=0.038$ ) as the most prominent factors for OS. Our study reveals the association of CD14-159 T allele with the presence of extranodal DLBCL and elevated CRP level at diagnosis. However, during the treatment T allele manifested protective role since patients with CT/TT genotypes less frequently experienced infections and early-treatment-related complications. In addition, TT genotype was associated with improved survival, but only in patients with extranodal disease.
\end{abstract}

Keywords: Diffuse large B-cell lymphoma, CD14, Genotyping

\section{INTRODUCTION}

Diffuse large B-cell lymphoma (DLBCL), a heterogeneous group of diseases, constitutes $30-58 \%$ of all non-Hodgkin lymphoma cases. ${ }^{1}$ In the majority of patients DLBCL is successfully treated with immunochemotherapy composed of rituximab, cyclophosphamide, doxorubicin, vincristine and prednisone (R-CHOP). However, most patients who failed R-CHOP have no true curative options and will ultimately die from lymphoma. Thus, there is a need for more effective front-line and salvage strategies. ${ }^{2}$ In the majority of cases, pattern of gene expression reflects the stage of lymphoid differentiation and classifies DLBCL as germinal center B cell (GCB) or activated B cell (ABC) subtype. This molecular distinction has prognostic implications (GCB subtype exhibits superior survival following R-CHOP than ABC subtype) and also reveals oncogenic signaling pathways that could be explored for novel therapeutic strategies. ${ }^{2-4}$ However, large body of evidences clearly indicates that both, intrinsic defects affecting the regulation of cell division and/or apoptosis as well as an altered survival-stimulating tumor environment are significant factors of pathogenesis and clinical progression. ${ }^{5,6}$ 
Thus, molecules included in innate immunity have been identified as candidates for effects on cancer, such as CD14 (cluster of differentiation 14).

The CD14 molecule is expressed on the surface of monocytes, macrophages, neutrophils and dendritic cells as a membrane-anchored form, but also exist as a soluble protein. It acts as a co-receptor for the detection of bacterial lipopolysaccharide (LPS). Soluble CD14 is capable to modulate cellular and humoral immune response by interacting directly with $\mathrm{T}$ and $\mathrm{B}$ lymphocytes. Increased levels of soluble CD14 were found in a wide variety of infectious and inflammatory diseases, but also in patients with NHL. ${ }^{7}$

There are several polymorphic sites in the CD14 gene, but CD14 -159C/T (rs2569190) in the promoter region is the most commonly studied. Previous studies demonstrated that the presence of $\mathrm{T}$ allele is associated with higher level of soluble CD148. The CD14 -159C/T polymorphism may be implicated in the development of gastric cancer in H. pylori - infected individuals ${ }^{9,10}$, gastric MALT lymphoma11 as well as childhood Hodgkin lymphoma. ${ }^{12}$

The aim of this study was to assess the association between CD14 -159 genotypes and DLBCL characteristics, clinical course and outcome, respectively. Since CD14 -159 T allele was associated with extranodal lymphoma, we also analyzed the impact of CD14 genotypes on outcome in this group of DLBCL patients.

\section{PATIENTS AND METHODS}

\section{Patients}

This study was approved by the Ethics committee of MMA, Belgrade. The study confirms the principles outlined in Helsinki Declaration.

The study included 114 patients (59 men and 55 women), aged 18 to 87 (median 52) with the newly diagnosed DLBCL ${ }^{13,14}$ who were diagnosed, treated and followed-up at the Clinic for hematology, MMA, Belgrade, Serbia. The patients with previous history of malignant disease or HIV-related DLBCL were not included in this study.
Diagnostic procedure included clinical examination, laboratory testing, bone marrow biopsy, chest radiograph and computer tomography of chest and abdomen. All patients received R-CHOP therapy (6-8 cycles). The follow-up period ranged from 2 to 135 (median 60) months.

\section{CD14 genotyping}

Blood was collected in EDTA tubes and stored at $-40^{\circ} \mathrm{C}$. DNA was extracted by a PureLinkTM Genomic DNA MiniKit (Invitrogen, Carlasbad, USA) according to the manufacturer's instructions.

$\mathrm{CD} 14-159 \mathrm{C} \rightarrow \mathrm{T}$ genotyping was performed by using PCR and restriction enzyme digestion with Hae III (Fermentas, Vilnius, Lithuania) as previously described. ${ }^{15}$

\section{Statistical Analysis}

The association between genotypes and clinical characteristics (age, sex, stage of disease, presence of extranodal sites, bulky disease, IPI score, absolute lymphocyte and absolute lymphocyte/ monocyte count), response to therapy, incidence of relapse, the presence of infections and early-treatment-related complications (neutropenia grade III/ IV, mucositis, acute renal failure, hart failure) was analyzed using the Pearson chi-squared test. Cuttof values for absolute lymphocyte count (1220 lymphocytes $/ \mathrm{mm}^{3}$ ) and absolute lymphocyte count/absolute monocyte count (2870) were determined by receiver operating curve (ROC) analysis.

The association between genotypes and markers of inflammation was analyzed by two-tailed t-test.

Relapse free survival (RFS) was calculated as the time from the start of treatment to relapse. Overallsurvival (OS) was defined as the time from the start of treatment to death or to date of last follow-up. Survival curves were generated using the method of Kaplan and Meier and compared by the logrank test. The most prominent factors for survival were determined by Cox proportional hazard regression (Wald test).

The $\mathrm{p}$ value $<0.05$ was considered statisticaly significant. 


\begin{tabular}{|c|c|c|c|c|}
\hline Patient/DLBCL characteristics & $(n=114)$ & $\begin{array}{l}\text { CD14 CT+TT } \\
\text { n (\%) }\end{array}$ & $\begin{array}{l}\text { CD14 CC } \\
\text { n (\%) }\end{array}$ & $\mathbf{p}$ \\
\hline Women & $55(48)$ & $10(18)$ & $45(82)$ & \\
\hline Man & $59(52)$ & $16(27)$ & $43(73)$ & \\
\hline$<60$ years & $79(69)$ & $19(24)$ & $60(76)$ & 0.632 \\
\hline$\geq 60$ years & $35(31)$ & $7(20)$ & $28(80)$ & \\
\hline IPI 0, 1, 2 & $75(66)$ & $18(24)$ & $57(76)$ & 0.671 \\
\hline IPI 3, 4, 5 & $39(34)$ & $8(21)$ & $31(79)$ & \\
\hline Clinical stage $\mid / / I$ & $60(53)$ & $14(23)$ & $46(77)$ & 0.888 \\
\hline Clinical stage III/IV & $54(47)$ & $12(22)$ & $42(78)$ & \\
\hline Extranodal disease & $80(70)$ & $13(16)$ & $67(84)$ & 0.01 \\
\hline No extranodal disease & $34(30)$ & $13(38)$ & $21(62)$ & \\
\hline Bulky disease & $53(46)$ & $12(23)$ & $41(77)$ & 1 \\
\hline No bulky disease & $61(54)$ & $14(23)$ & $47(77)$ & \\
\hline Absolute lymphocyte count & & & & 0.475 \\
\hline$<1220 / \mathrm{mm}^{3}$ & $72(66)$ & $18(25)$ & $54(75)$ & \\
\hline$\geq 1220 / \mathrm{mm}^{3}$ & $37(34)$ & $7(19)$ & $30(81)$ & \\
\hline Abs. lymph/abs. monocyte count & & & & 0.386 \\
\hline$<2870 / \mathrm{mm}^{3}$ & $66(61)$ & $17(26)$ & $49(74)$ & \\
\hline$\geq 2870 / \mathrm{mm}^{3}$ & $43(39)$ & $8(19)$ & $35(81)$ & \\
\hline
\end{tabular}

Table 1 presents the association between genotypes (CC vs CT+TT) and clinical characteristics (age, sex, stage of disease, presence of extranodal sites, bulky disease, IPI score, absolute lymphocyte and absolute lymphocyte/monocyte count).

Obtained frequencies of CD14 -159 genotypes were as follows: $23 \%$ of CC, $41 \%$ of CT and $36 \%$ of TT (Hardy-Weinberg equilibrium, $\mathrm{p}=0.883$ ).

Statistically significant association between CD14 genotypes and clinical characteristics was found only for extranodal disease $(\mathrm{p}=0.01)$. Compared to $\mathrm{CC}$ homozygotes, carriers of T allele (CT/TT genotypes) were more likely to have extranodal disease (OR 3.191, 95 \% CI: 1.282 - 7.94). The most common extranodal site was stomach (23/80).

Pretreatment values of C-reactive protein (CRP) were available for 101 patients. CRP values ranged $2.5-122 \mathrm{mg} / \mathrm{l}$ (average 24) in patients with CC genotype $(\mathrm{n}=24)$ and $2.2-266 \mathrm{mg} / \mathrm{l}$ (average 39.5) in patients with CT/TT genotype $(\mathrm{n}=77)$. The association between genotypes and CRP values was of marginal statistical significance (one-tailed ttest $\mathrm{p}=0.038$; two-tailed t-test $\mathrm{p}=0.078$ ). Values of other inflammatory markers (sedimentation of erythrocytes and fibrinogen) were not associated with CD14 genotypes.

\section{CD14 genotypes and course of DLBCL}

Of 114 DLBCL patients, 98 (86\%) responded to RCHOP; 69 (61\%) achieved complete remission and $29(25 \%)$ had partial response. Disease progression during the therapy was present in $16(14 \%)$ patients. Overall, 28/98 patients had disease recurrence and 34/114 (30\%) died. CD14 genotypes (CC vs $\mathrm{CT}+\mathrm{TT})$ were not associated with response to therapy, incidence of relapse and outcome.

Infections during the therapy were present in 29 (25\%) patients. Infections were more common in patients with CC than in patients with CT/TT genotypes (39\% vs $22 \%$ ). The association between genotypes and infections was of marginal statistical significance $(p=0.083)$. Early-treatment-related complications [present in $31(27 \%)$ patients] were observed in $42 \%$ of patients with CC and in $24 \%$ of patients with CT/TT genotypes $(\mathrm{p}=0.079)$.

In the group of patients with DLBCL, CD14 -159 genotypes were not associated with the duration of RFS $(p=0.351)$ and OS $(p=0.485)$ (Figure 1). In this group of patients, OS was affected by IPI score $(\mathrm{p}<0.001)$, clinical stage $(\mathrm{p}<0.001)$, absolute lymphocyte count ( $\mathrm{p}=0.001)$ and absolute lymphocyte/ absolute monocyte count $(\mathrm{p}<0.001)$. Multivariate analysis revealed IPI score $(\mathrm{p}=0.006)$ as the most prominent factor for OS. 

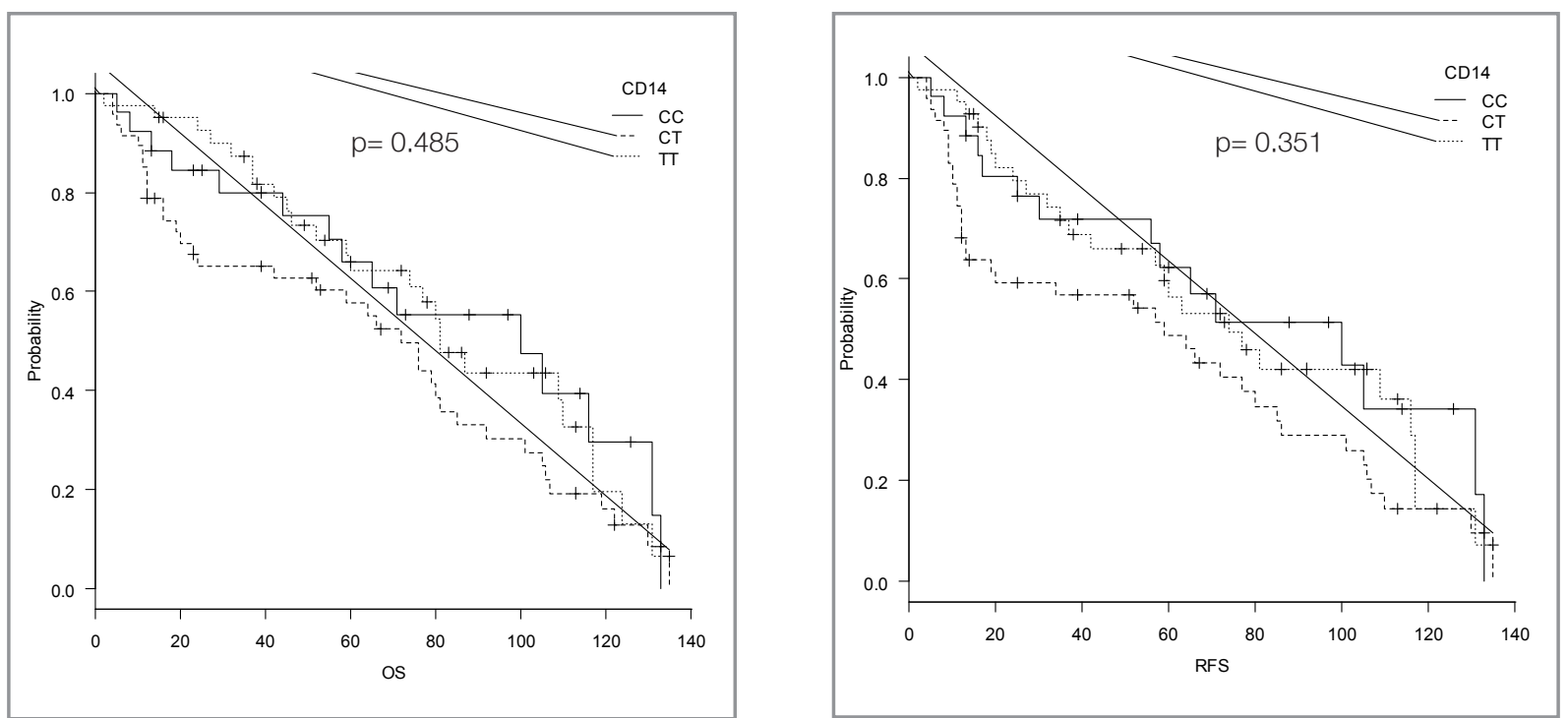

Figure 1. A: Overall survival in DLBCL patients with different CD14 -159 genotypes; B: Relapse free survival in DLBCL patients with different CD14 -159 genotypes

In the group of patients with extranodal DLBCL, superior OS was observed in patients with TT genotype (3-year OS of $84 \%$ ) than in those with CC (3-year OS of 77\%) and CT genotype (3-year OS of $68 \%$ ). However, statistical analysis didn't show significant association between genotypes and OS $(p=0.122)$. When OS was compared between patients with TT (3-year OS of 84\%) and CC/CT genotypes (3-year OS of 70\%), statistical analysis showed significant association between genotypes and OS $(\mathrm{p}=0.049)$. Similar pattern was observed for RFS, (CC vs CT vs TT, $\mathrm{p}=0.045$; CC/CT vs TT, $\mathrm{p}=0.018)$. OS of patients with extranodal DLBCL was also affected with IPI score $(p<0.001)$, clinical stage $(\mathrm{p}=0.015)$, the presence of bulky disease $(\mathrm{p}=$ $0.032)$, absolute lymphocyte count $(\mathrm{p}=0.01)$ and absolute lymphocyte/ absolute monocyte count $(\mathrm{p}=$ 0.001). In multivariate analysis IPI [hazard ratio (HR) 2.422, 95\% CI: 1.114-5.264; $\mathrm{p}=0.026$ ) and CD14 TT genotype (HR 0.503, $95 \%$ CI 0.262$0.963 ; p=0.038$ ) were the most prominent factors for OS in patients with extranodal DLBCL (Table 2). Figure 2 presents the association of $\mathrm{CD} 14$ genotypes and survival.

\section{DISCUSSION}

CD14 exerts multiple roles in innate immune cells with a wide range of consequences in physiologic and pathologic conditions.
The present study on DLBCL reports the association between CD14 -159 genotypes and the presence of extranodal disease. Extranodal involvement was more frequent in patients with high-producing $\mathrm{T}$ allele (CT and TT) genotypes. One quarter of our patients with extranodal disease had gastric DLCBL. The role of CD14 in gastric carcinogenesis and invasion of gastric cancer cells was previously demonstrated. ${ }^{16}$ Meta-analysis performed by Wang and coworkers suggested that CD14 polymorphism may increase the risk of gastric cancer in H. pylori-infected individuals9. Considering lymphoma, Türe-Özdemir and coworkers found association between CD14 $\mathrm{T}$ allele and development of gastric MALT lymphoma11. As reported, CD14 together with TLR binds to bacterial (H. pylori or other) LPS and consequently activates the release of cytokines which may establish chronic inflammation and contribute to gastric carcinogenesis 11 . We can assume that suggested mechanism of carcinogenesis is also applicable for development of gastric DLBCL. In the review on CD14 role in host protection against infection, Zanoni and Granucci outlined that the contribution of CD14 to infection control depends both on the microorganism and the site of infection. ${ }^{17}$ To explain the role of CD14 in the development of extranodal DLBCL, better understanding of regional influence exerted by CD14 as well as the functions of CD14 in nonhematopoietic cells is needed. 
International Journal of Hematology and Oncology

Table 2. Multiparametric analysis for the most prominent factors for survival in patients with extranodal DLBCL

\begin{tabular}{|llll|}
\hline & Hazard ratio & $\mathbf{9 5 \%} \mathbf{C l}$ & $\mathbf{p}^{\mathbf{a}}$ \\
\hline Bulky disease & 1.047 & $0.543-2.019$ & 0.89 \\
Clinical stage III or IV & 0.947 & $0.405-2.217$ & 0.901 \\
IPI 3, 4 or 5 & 2.422 & $1.114-5.264$ & 0.026 \\
Absolute lymphocyte count $>1220 \mathrm{ly} / \mathrm{mm}^{3}$ & 0.859 & $0.255-2.89$ & 0.806 \\
Abs. lymph/abs. monoc count $>2870 / \mathrm{mm}^{3}$ & 0.609 & $0.203-1.827$ & 0.376 \\
CD14 -159 TT genotype & 0.503 & $0.262-0.963$ & 0.038 \\
\hline
\end{tabular}

Soluble form of CD14 is detectable in the blood of healthy individuals. Increased levels of soluble CD14 seem to be associated with a variety of inflammatory diseases. Baseggio and coworkers reported that NHL patients had significantly higher concentrations of CD14 in the blood than healthy
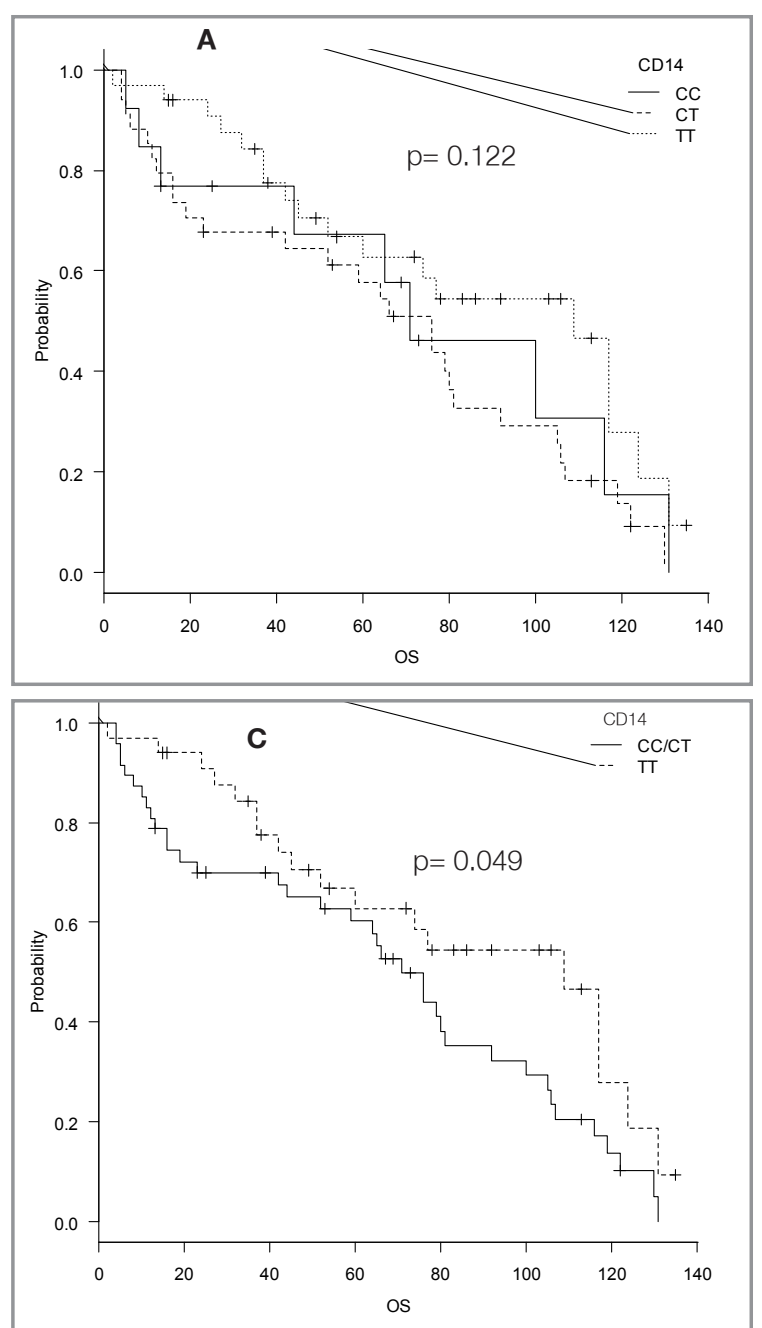

individuals, while patients with aggressive DLBCL or Burkitt lymphoma had a slightly higher CD14 values than patients with indolent lymphoma. In addition, soluble CD14 levels were significantly correlated with CRP and TNF levels, reflecting the cytokine-mediated inflammation. ${ }^{7}$ In our study,
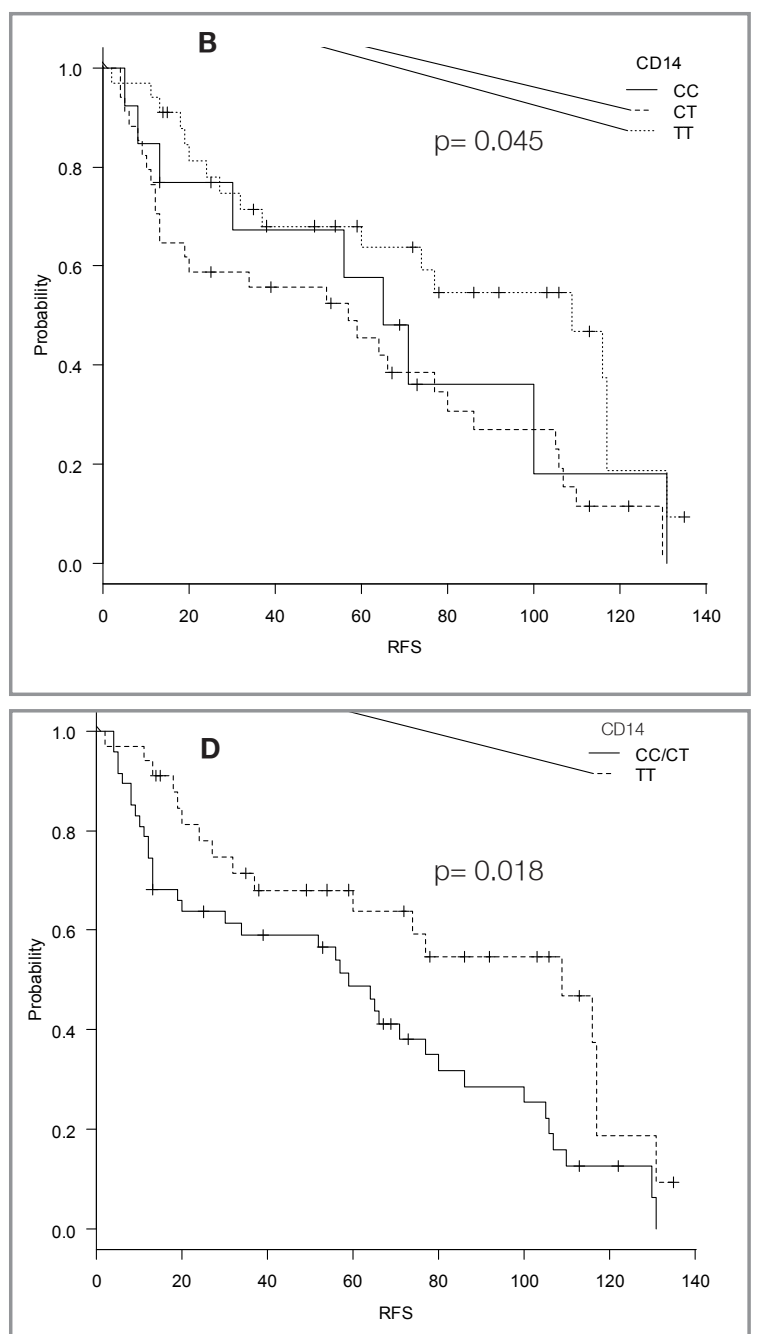

Figure 2. A: Overall survival in patients with extranodal DLBCL in regard to different CD14 -159 genotypes; B: Relapse free survival in patients with extranodal DLBCL in regard to different CD14 -159 genotypes; C: Overall survival in patients with extranodal DLBCL in regard to CD14 -159 genotypes (CC/CT vs TT); D: Relapse free survival in patients with extranodal DLBCL in regard to CD14 -159 genotypes (CC/CT vs TT) 
patients with $\mathrm{CD} 14$ high-producing $\mathrm{T}$ allele (CT/ TT genotypes) had higher CRP values at diagnosis than patients with CC genotype (marginal statistical significance). Previous reports demonstrated high CRP levels at diagnosis of DLBCL as an independent poor prognostic factor for clinical outcome. $^{18}$

The majority of studies reported association between CD14 overexpression and frequent/prolonged infection, indicating a functional correlation of CD14 overexpression with a dysfunctional innate immune system which may promote tumorigenesis. ${ }^{19,20}$ Our findings of association between high-producing $\mathrm{CD} 14 \mathrm{~T}$ allele with extranodal DLBCL and elevated CRP values at diagnosis are concordant with these reports. However, our patients with CD14 $\mathrm{T}$ allele (CT/TT genotypes) less frequently experienced treatment-related infections as well as early-treatment-related complications, both previously reported as unfavorable clinical events. ${ }^{21,22}$

In our group of 114 patients with DLBCL, CD14 -159 genotypes didn't influence RFS and OS. However, in the group of patients with extranodal disease, homozygous carriers of high-producing $\mathrm{T}$ allele (TT genotype) had superior RFS and OS. Previous reports about the association between CD14 genotypes/production and survival in cancer patients brought different conclusions. Thus, overexpression of CD14 on tumor cells was associated with progression of bladder cancer and poor outcome. ${ }^{23}$ In the article of Seiffert and coworkers, soluble CD14 has been identified as a novel survival factor for chronic lymphocytic leukemia cells. ${ }^{24}$ However, analyzing the gene expression in primary tumors at diagnosis from children with metastatic neuroblastoma, Stigliani and coworkers reported superior event free survival and OS in patients with high levels of CD14 mRNA, whereas low levels of CD14 mRNA identified patients with poor survival. The authors suggested that cells (macrophages and dendritic cells) expressing CD14 may be responsible for the activation of $\mathrm{T}$ lymphocytes, which in turn limited tumor progression. ${ }^{25}$ The studies on association between CD14 genotypes and outcome in cancer patients are rare. Clinical impact of CD14 genotypes is often studied in critically ill patients, but obtained results were inconsistent. Despite frequent findings of poor outcome in patients with CD14 -159 TT genotype, Mansur and coworkers reported improved survival in those patients. ${ }^{26}$ Similarly, superior survival in critically ill patients after burn injury was noticed in CD14 -159 TT carriers. ${ }^{27}$

In summary, our study indicates the association of CD14 -159 T allele and the presence of extranodal DLBCL and elevated CRP at diagnosis, both previously established as unfavorable clinical parameters. However, during the treatment protective role of T allele was manifested since patients with CT/ TT genotypes less frequently experienced treatment-related infections as well as early-treatmentrelated complications. In addition, TT genotype was associated with improved survival, but only in patients with extranodal disease. Considering this study as well as previous reports, we can conclude that overexpression of CD14 (or the presence of high producing allele/genotype) may lead to different outcomes. The link between CD14 overexpression and chronic inflammation/tumorigenesis was previously established. However, the role of CD14 overexpression and inflammation in clinical course of DLBCL remains to be resolved.

\section{Funding:}

This work was supported by the University of Defense - Faculty of Medicine, Belgrade, Serbia; under Grant [MFVMA/F19/12-19 and MFVMA/12/16-18].

\section{REFERENCES}

1. Tilly H, Vitolo U, Walewski J, et al. Diffuse large B-cell lymphoma (DLBCL): ESMO Clinical Practice Guidelines for diagnosis, treatment and follow-up. Ann Oncol 23 (Suppl 7): vii78-vii82, 2012.

2. Sehn LH, Gascoyne RD. Diffuse large B-cell lymphoma: optimizing outcome in the context of clinical and biologic heterogeneity. Blood 125: 22-32, 2015.

3. Rosenwald A, Wright G, Chan WC, et al. The use of molecular profiling to predict survival after chemotherapy for diffuse large-B-cell lymphoma. N Engl J Med 346: 1937-47, 2002.

4. Pasqualucci L. The genetic basis of diffuse large B-cell lymphoma. Curr Opin Hematol 20: 336-344, 2013. 
5. Pattabiraman DR, Weinberg RA. Tackling the cancer stem cells - what challenges do they pose? Nature Rev. Drug Discov 13: 498-512, 2014

6. Wu C, Wu X, Liu X, et al. Prognostic Significance of Monocytes and Monocytic Myeloid-Derived Suppressor Cells in Diffuse Large B-Cell Lymphoma Treated with R-CHOP. Cell Physiol Biochem 39: 521-530, 2016.

7. Baseggio L, Charlot C, Picollet J, et al. High circulating tumor necrosis factor levels correlate with increased evels of soluble CD14 in patients with non-Hodgkin's lymphoma. Haematologica 86: 774-775, 2001.

8. LeVan TD, Bloom JW, Bailey TJ, et al. A common single nucleotide polymorphism in the CD14 promoter decreases the affinity of Sp protein binding and enhances transcriptional activity. J Immunol 167: 5838-5844, 2001.

9. Wang J, Guo X, Yu S, et al. Association between CD14 Gene Polymorphisms and Cancer Risk: A Meta-Analysis. PLoS One 9: e100122, 2014.

10. Zhao D, Sun T, Zhang X, et al. Role of CD14 promoter polymorphisms in Helicobacter pylori infection--related gastric carcinoma. Clin Cancer Res 13: 2362-2368, 2007.

11. Türe-Ozdemir F, Gazouli M, Tzivras M, et al. Association of polymorphisms of NOD2, TLR4 and CD14 genes with susceptibility to gastric mucosa-associated lymphoid tissue lymphoma. Anticancer Res 28(6A): 3697-700, 2008.

12. Andrie E, Michos A, Kalampoki V, et al. Genetic variants in immunoregulatory genes and risk for childhood lymphomas. Eur J Haematol 83: 334-342, 2009.

13. Gatter KC, Warnke RA. Diffuse large B-cell lymphoma. In: Jaffe ES, Harris NL, Stein H, Vardiman JW (eds) World Health Organization Classification of Tumours. Pathology and genetics of tumours of haematopoietic and lymphoid tissues. Lyon, IARC Press, 2001: 171-174.

14. Campo E, Swerdlow SH, Harris NL, et al. The 2008 WHO classification of lymphoid neoplasms and beyond: evolving concepts and practical applications. Blood 117: 5019-5032, 2011.

15. Gibot S, Cariou A, Drouet L, et al. Association between a genomic polymorphism within the CD14 locus and septic shock susceptibility and mortality rate. Crit Care Med 30: 969-973, 2002.

16. Li K, Dan Z, Hu X, et al. CD14 regulates gastric cancer cell epithelial mesenchymal transition and invasion in vitro. Oncol Rep 30: 2725-2732, 2013.

17. Zanoni I, Granucci F. Role of CD14 in host protection against infections and in metabolism regulation. Front Cell Infect Microbiol 3: 32, 2013.

18. Troppan KT, Schlick K, Deutsch A, et al. C-reactive protein level is a prognostic indicator for survival and improves the predictive ability of the R-IPI score in diffuse large B-cell lymphoma patients. Br J Cancer 111: 55-60, 2014.
19. Keerthivasan G, Mei Y, Zhao B, et al. Aberrant overexpression of CD14 on granulocytes sensitizes the innate immune response in mDia1 heterozygous del(5q) MDS. Blood 124: 780-790, 2014.

20. Hu Y, Yoshida K, Cologne JB, et al. CD14 and IL18 gene polymorphisms associated with colorectal cancer subsite risks among atomic bomb survivors. Hum Genome Var 2: 15035, 2015.

21. Pole JD, Gibson P, Ethier MC, et al. Evaluation of treatmentrelated mortality among paediatric cancer deaths: a population based analysis. Br J Cancer 116: 540-545, 2017.

22. Jung $\mathrm{YH}$, Woo IS, Han CW. Clinical characteristics and outcomes in diffuse large B cell lymphoma patients aged 70 years and older: a single-center experience with a literature review. Korean J Intern Med 30: 684-693, 2015.

23. .Cheah MT, Chen JY, Sahoo D, et al. CD14-expressing cancer cells establish the inflammatory and proliferative tumor microenvironment in bladder cancer. Proc Natl Acad Sci U S A 112: 4725-430, 2015

24. Seiffert M, Schulz A, Ohl S, et al. Soluble CD14 is a novel monocyte-derived survival factor for chronic lymphocytic leukemia cells, which is induced by CLL cells in vitro and present at abnormally high levels in vivo. Blood 116: 4223-4230, 2010.

25. Stigliani S, Croce M, Morandi F, et al. Expression of FOXP3, CD14, and ARG1 in Neuroblastoma Tumor Tissue from High-Risk Patients Predicts Event-Free and Overall Survival. Biomed Res Int 2015: 347867, 2015.

26. Mansur A, Liese B, Steinau M, et al. The CD14 rs2569190 $\Pi$ Genotype Is Associated with an Improved 30-Day Survival in Patients with Sepsis: A Prospective Observational Cohort Study. PLoS One 2015 10(5): e0127761.

27. Barber RC, Aragaki CC, Chang LY, et al. CD14-159 C allele is associated with increased risk of mortality after burn injury. Shock 27: 232-237, 2007.

\section{Correspondence:}

Cikota-Aleksic Bojana, Ph.D.

Institute of Medical Research, Military Medical Academy

Crnotravska 17

11000 Belgrade, SERBIA

Tel: +381113608339

Cell phone: +381641430237

Fax: +381112662 722

e-mail: cbojana@ptt.rs; cbojana@yahoo.com 